\title{
Research on time difference detection algorithm based on combination of GNSS and PPP
}

\author{
Jun Yang ${ }^{1}$, Ziwen Zhang ${ }^{2 *}$, Yijun Liu' ${ }^{2}$ Zuoteng $\mathrm{Xu}^{2}$, Haowen Chen², Wende Zhang ${ }^{2}$ and Yuqiang Chen ${ }^{3}$
}

\begin{abstract}
With the gradual increase in the number of GNSS systems and the improvement of functions, in addition to the singlesystem navigation and timing service, the integrated navigation and positioning service among multiple systems can provide users with more accurate and stable positioning results, arousing more attention from the workers in GNSS field. Compatibility and interoperability among different systems has become a trend in the development of GNSS. Compatibility and interoperability between systems require a uniform time scale. Therefore, the measurement and forecasting of time deviations in GNSS systems is particularly important. This paper first studies the multi-system fusion location model and proposes an adaptive GNSS fusion PPP algorithm based on parameter equivalent reduction. Then, the method of fusion PPP is used to monitor the time difference of GNSS. Finally, the effectiveness of the improved algorithm and time difference monitoring method is verified by practical examples.
\end{abstract}

Keywords: GNSS, PPP algorithm, Pseudo-time difference, Time difference monitoring

\section{Introduction}

The official launching of China's Beidou second-generation satellite navigation system indicates that the number of global satellite navigation and positioning systems (GNSS) currently providing services has increased from two to three. The number of GNSS working satellites has exceeded 70. In the next 5 to 10 years, with the completion of the EU Galileo system, the total number of GNSS satellites will exceed 100, and the vast majority are modern multi-frequency operational satellites. GNSS has entered a new chapter of multi-constellation and multi-frequency [1]. With the modernization of GNSS, the timing accuracy of multi-system integrated navigation positioning is also increasing. The development characteristics of GNSS have also gradually changed from the single GPS positioning model that initially focuses on post-processing relative positioning to the multi-system GNSS fusion absolute positioning with fast real-time and high-frequency observations. Among them, the rapid and flexible precision point positioning technology

\footnotetext{
*Correspondence: zhzw2018@163.com

${ }^{2}$ School of Information Engineering, Guangdong University of Technology, Guangzhou 510006, China

Full list of author information is available at the end of the article
}

(PPP) is undoubtedly one of the most promising application technologies [2].

The accuracy and stability of real-time fast positioning will be greatly improved by using the fusion and positioning of GNSS multi-system. In recent years, the research results of GNSS fusion PPP both at home and abroad are more abundant, mainly concentrated in the [3] GPS/GLONASS fusion. For example, Dr. Kuang of JPL proposed that the precision orbit of GLONASS single star or constellation could be determined based on the space-time system of the GPS system, and the orbit determination accuracy of GLONASS satellite was increased to $15 \mathrm{~cm}$ level by this method. Based on the global dual mode receiver, Ignacio realizes the joint orbit determination method of GPS and GLONASS and analyzes the signal delay of the multi-mode system in different receivers in detail. Based on the ROCK light pressure model, ESA established the GLONASS on-orbit satellite solar photovoltaic model. These research results mainly demonstrate the accuracy and reliability advantages brought by fusion positioning and seldom involve the fusion algorithm itself and the distribution of the contribution ratio between different systems [4]. 


\subsection{Related work}

Although the study of GNSS in the time and frequency domain has been more than 30 years old, the study of time difference monitoring between systems using the GNSS technology method is still at a preliminary stage of research. With the gradual improvement of China's COMPASS system, it becomes more and more urgent to realize the compatibility and interoperability between the system and other GNSS systems [5]. At present, there are not many open researches on the time difference monitoring of GNSS systems at home and abroad, mainly focusing on GPS and Galileo and GPS and COMPASS. In recent years, the number of available GNSS systems and the number of available satellites has gradually increased, and real-time high-frequency data of terrestrial receivers have been widely used. In this context, for real-time mass data under multi-mode GNSS systems, if the traditional PPP fusion algorithm is used, all the GNSS observations are unified to form the observation equation for unified solution, and the computational load is bound to increase exponentially. At the same time, this kind of overall solution is also difficult to realize the adjustment of the weights of the adaptive contribution between different systems, which affects the operational efficiency and precision and reliability of the fusion PPP positioning [6].

The GNSS fusion sequential PPP algorithm based on the principle of parameter equivalence reduction can solve the abovementioned overall problem of low efficiency. This algorithm decomposes the multi-mode PPP integral fusion solution into individual single-system independent parallel solutions. The equations of the overlapped parametric equations among different systems are equivalently reduced by using the normal equations constructed by the single system, and the fusion solutions can be directly obtained by superposition. The main advantage of the new algorithm is to improve its computational efficiency. With the increase of the number of fusion systems, the computational load of traditional algorithms increases exponentially, and the new algorithm could improve it to a linear growth. In addition, this paper also proposes an adaptive fusion method that balances the contribution weight ratios of different systems by using the posttest difference factor. Finally, the effectiveness and accuracy of the proposed algorithm are verified by the comparison of actual data operation time and dynamic and static positioning tests.

\section{Methods}

\subsection{Single-system PPP algorithm based on parameter reduction}

The parameters in the single-system observation equation are divided into two categories. The classified observation equation can be written as the following block matrix form:

$$
V=A_{1} X_{1}+A_{2} X_{2}-L, \quad P
$$

Among them, $X_{1}$ is a parameter that changes with time and mainly refers to the receiver clock offset parameter in PPP. $X_{2}$ is a parameter that does not change over time and mainly refers to coordinate parameters, tropospheric parameters, and ambiguity parameters in PPP. What needs to be explained here is that the coordinate parameters between epochs during dynamic PPP positioning are also changed. It is not possible to carry out inheritance directly, and it can be forecasted by filtering the state equation [7].

The partitioned equation obtained by formula (1) is as follows:

$$
\left[\begin{array}{ll}
B_{11} & B_{12} \\
B_{21} & B_{22}
\end{array}\right]\left[\begin{array}{l}
X_{1} \\
X_{2}
\end{array}\right]=\left[\begin{array}{l}
C_{11} \\
C_{22}
\end{array}\right]
$$

wherein $B_{11}=A_{1}^{T} P A_{1}, B_{12}=A_{1}^{T} P A_{2}, B_{21}=B_{12}^{T}, B_{22}=A_{2}^{T}$ $P A_{2}, C_{11}=A_{1}^{T} P L, C_{22}=A_{2}^{T} P L$.

Carry out the equivalent reduction of the parameters of formula (2) and it could be obtained that:

$$
\left[\begin{array}{cc}
B_{11} & B_{12} \\
0 & B_{2}
\end{array}\right]\left[\begin{array}{l}
X_{1} \\
X_{2}
\end{array}\right]=\left[\begin{array}{l}
C_{11} \\
R_{2}
\end{array}\right]
$$

wherein $\mathrm{B}_{2}=\mathrm{B}_{22}-\mathrm{B}_{21} \mathrm{~B}_{11}^{-1} \mathrm{~B}_{12}, R_{2}=C_{22}-B_{21} B_{11}^{-1} C_{11}$.

For formula (3), $X_{2}$ is solved by the second formula, and then $X_{1}$ is returned through the first formula, that is

$$
\begin{aligned}
& B_{2} X_{2}=R_{2} \\
& B_{11} X_{1}=C_{11}-B_{12} X_{2}
\end{aligned}
$$

That is, the single system PPP recurrence calculation formula for the $i$ th epoch can be obtained as follows:

$$
\left\{\begin{aligned}
\hat{B}_{2}^{\mathrm{i}}=B_{2}^{\mathrm{i}}+\hat{B}_{2}^{\mathrm{i}-1} \\
\hat{R}_{2}^{\mathrm{i}}=R_{2}^{\mathrm{i}}+\hat{R}_{2}^{\mathrm{i}-1} \\
X_{2}=\left(\hat{B}_{2}^{\mathrm{i}}\right)^{-1} \hat{R}_{2}^{\mathrm{i}} \\
X_{1}^{\mathrm{i}}=\left(B_{11}^{\mathrm{i}}\right)^{-1}\left(C_{11}^{\mathrm{i}}-B_{12}^{\mathrm{i}} X_{2}\right) \\
Q_{X_{2}}=\left(\hat{B}_{2}^{\mathrm{i}}\right)^{-1} \\
Q_{X_{2}^{\mathrm{i}}=}\left(B_{11}^{\mathrm{i}}-B_{12}^{\mathrm{i}}\left(B_{22(\text { new })}^{\mathrm{i}}\right)^{-1} B_{21}^{\mathrm{i}}\right)^{-1}, \\
\quad B_{22(\text { new })}^{\mathrm{i}}=\hat{B}_{2}^{\mathrm{i}}+B_{21}^{\mathrm{i}}\left(B_{11}^{\mathrm{i}}\right)^{-1} B_{12}^{\mathrm{i}}
\end{aligned}\right.
$$

Through the recursive calculation of formula (12), the single-system static PPP sequential solution based on parameter reduction can be realized. 


\subsection{Multi-system fusion PPP algorithm based on}

\section{parameter reduction}

For multi-system fusion positioning, the fixed parameters $X_{2}$ in the observation equations of each single system can be divided into two categories[8], namely $X_{2}$ $=\left[\begin{array}{ll}Y_{1} & Y_{2}\end{array}\right]^{T}$. One is the overlapping parameters $Y_{1}$ between different systems, such as position and troposphere parameters. The other is non-overlapping parameters $Y_{2}$ that represent ambiguities and so on.

Therefore, the $B_{2}$ and $R_{2}$ obtained by the reduction in equation (4) is rewritten into the following block matrix form:

$$
B_{2}=\left[\begin{array}{ll}
M_{11} & M_{12} \\
M_{21} & M_{22}
\end{array}\right], \quad R_{2}=\left[\begin{array}{l}
R_{11} \\
R_{22}
\end{array}\right]
$$

Formula (4) is written in the following matrix according to extension of $Y_{1}$ and $Y_{2}$ :

$$
\left[\begin{array}{ll}
M_{11} & M_{12} \\
M_{21} & M_{22}
\end{array}\right]\left[\begin{array}{l}
Y_{1} \\
Y_{2}
\end{array}\right]=\left[\begin{array}{l}
R_{11} \\
R_{22}
\end{array}\right]
$$

It could be obtained from formula (8):

$$
\left[\begin{array}{cc}
M_{1} & 0 \\
M_{21} & M_{22}
\end{array}\right]\left[\begin{array}{l}
Y_{1} \\
Y_{2}
\end{array}\right]=\left[\begin{array}{c}
R_{1} \\
R_{22}
\end{array}\right]
$$

wherein $M_{1}=M_{11}-M_{12} M_{22}^{-1} M_{21}, R_{1}=R_{11}-M_{12} M_{22}^{-1} R_{22}$. And then:

$$
M_{1} Y_{1}=R_{1}
$$

For GPS, GLONASS, COMPASS, etc., the common parameter in the parameters to be evaluated is $Y_{1}$. Therefore, the results of the overlapping parameter equation (Eq. (10)) for each system can be obtained separately. The equations are as follows:

$$
\begin{aligned}
M_{1}^{G} Y_{1} & =R_{1}^{G}, M_{1}^{R} Y_{1}=R_{1}^{R}, M_{1}^{C} Y_{1} \\
& =R_{1}^{C}, \quad M_{1}^{E} Y_{1}=R_{1}^{E}, \ldots,
\end{aligned}
$$

In the above formulae, the superscripts $G, R, C$, and $E$ represent the GPS system, the GLONASS system, the COMPASS system, and the GALILEO system, respectively.

Therefore, the equation for solving the GNSS multi-system fusion PPP solution is as follows:

$$
\sum_{k=1}^{m} M_{1}^{\mathrm{k}} Y_{1}=\sum_{k=1}^{m} R_{1}^{k}
$$

Among them, $M$ represents the number of satellite navigation systems.

After the value of $Y_{1}$ is calculated, the value of $Y_{2}$ can be recovered by the formula (8) or (9); thus, the value of $X_{2}$ could be obtained and then recovery the value of $X_{1}$, and then the fusion PPP is solved. The multi-system PPP fusion formula for the $i$ th epoch is as follows:

$$
\left\{\begin{array}{l}
\hat{B}_{2 \text { (new) }}^{i}=\hat{B}_{2}^{\mathrm{i}}+\left[\begin{array}{cc}
\sum_{k=1, k_{i} l}^{m} M_{1}^{k} & 0 \\
0 & 0
\end{array}\right] \\
\hat{R}_{2 \text { (new) }}^{i}=\hat{R}_{2}^{i}+\left[\begin{array}{c}
\sum_{k=1, k_{i} l}^{m} R_{1}^{k} \\
0
\end{array}\right] \\
X_{2}=\left(\hat{B}_{2(\text { new })}^{i}\right)^{-1} \hat{R}_{2(\text { new })}^{i} \\
X_{1}^{i}=\left(B_{11}^{i}\right)^{-1}\left(C_{11}^{i}-B_{12}^{i} X_{2}\right) \\
Q_{X_{2}}=\left(\hat{B}_{2(\text { new })}^{i}\right)^{-1} \\
Q_{X_{2}^{i}}=\left(B_{11}^{i}-B_{12}^{i}\left(B_{22(\text { new })}^{i}\right)^{-1} B_{21}^{i}\right)^{-1} \\
B_{22 \text { (new })}^{i}=\hat{B}_{2}^{i}+B_{21}^{i}\left(B_{11}^{i}\right)^{-1} B_{12}^{i}
\end{array}\right.
$$

In the equation (13), $\sum_{k=1, k_{i} l}^{m} M_{1}^{k}$ and $\sum_{k=1, k_{i} l}^{m} R_{1}^{k}$ represents the sum of the normal equation observation matrix of systems other than the own system.

\subsection{An adaptive PPP fusion algorithm based on posttesting error factor}

The current three GNSS navigation systems are capable of providing four or more visual satellites for user navigation and positioning in real time or over the world. This provides us with a very valuable prior information in the process of processing the fusion data. That is, a single system can obtain independent overlapped parameter values and their accuracy information, making the adaptive fusion PPP localization possible in data processing.

Therefore, in this paper, the adaptive factor is determined by using the posterior variance value of the overlapping parameters obtained by the single system.

The adaptive factor $a_{k}$ of the $\mathrm{k}$ system is set as follows:

$$
a_{k}=\left[\begin{array}{cccc}
1 / \sigma_{x_{k}}^{2} & 0 & 0 & 0 \\
0 & 1 / \sigma_{y_{k}}^{2} & 0 & 0 \\
0 & 0 & 1 / \sigma_{z_{k}}^{2} & 0 \\
0 & 0 & 0 & 1 / \sigma_{T_{k}}^{2}
\end{array}\right]
$$

Among them, $\sigma_{x_{k}}^{2}, \sigma_{y_{k}}^{2}, \quad \sigma_{z_{k}}^{2}$ and $\sigma_{T_{k}}^{2}$ are obtained through multiplying the posterior variance $\sigma_{0}^{2}$ of the single system by the diagonal elements of the corresponding parameter variance matrix $Q$. Formula (14) is a classification adaptive factor form which is classified and adjusted according to different parameters. In actual work, we can also directly take the square root of the sum of the variances of the four parameters to solve the overall single factor. The calculation formula is as follows: 


$$
a_{k}=\frac{1}{\sqrt{\left(\sigma_{x_{k}}^{2}+\sigma_{y_{k}}^{2}+\sigma_{z_{k}}^{2}+\sigma_{T_{k}}^{2}\right)}}
$$

The subsequent example used in this paper is the single factor adjustment method shown in (15).

The formula for solving the normal equation of the adaptive fusion PPP overlap parameter can be obtained as follows:

$$
\sum_{k=1}^{m} a_{k} M_{1}^{k} Y_{1}=\sum_{k=1}^{m} a_{k} R_{1}^{k}
$$

Through the above equation, when carrying out the fusion positioning of different GNSS system, the size of the contribution of the system to the fusion solution can be adaptively adjusted according to the single system's internal accuracy index, so as to avoid serious influence on the fusion solution when a system is grossly poor. This improves the accuracy and stability of multi-system fusion solutions.

The parameter reduction method in this paper can be extended to the research work of GNSS precision satellite clock error correction, non-differential baseline network adjustment, and GNSS multi-mode time difference monitoring, which can effectively improve its work efficiency. The feasibility and accuracy of the fusion PPP method in this section for GNSS time difference monitoring will be discussed below.

\section{Monitoring and analysis of GNSS time difference based on fusion PPP model}

\subsection{Concept of GNSS time difference and conventional} monitoring methods

There is a system deviation between the time systems of different GNSS navigation systems, known as time differences. Taking the GPS/GLONASS time difference as an example, there is the following transformation between GPST and GLOT:

$$
\mathrm{GPST}=\mathrm{GLOT}+\mathrm{\tau c}+\mathrm{\tau u}+\mathrm{\tau g}
$$

In this, $\tau c$ represents the time difference between the GLOT and the UTC (SU). Tu represents the time difference between UTC (SU) and international UTC. tg represents the time difference between international UTC and GPST. The time difference between GPST and GLOT is the sum of the above three.

At present, the GPS's broadcast ephemeris provides a linear time difference conversion model coefficient from GPST to UTC (USNO). The GLONASS broadcast ephemeris provides a time difference between GLOT and UTC (SU). Therefore, as long as the time comparison between UTC (USNO) and UTC (SU) is obtained, the GPS/GLONASS time difference can be obtained.
The time comparison between UTC (USNO) and UTC (SU) can be obtained by direct comparison of the master stations or their comparison with international UTC or International Atomic Time (TAI). A brief process for obtaining a time difference through a time comparison technique at the end of the system is shown in the diagram in Fig. 1.

The above time difference acquisition method has the advantages of high precision and stable and reliable time difference. It is one of the main ways to obtain the international GNSS TDOA. However, this time difference acquisition method is usually performed on the main control station of the GNSS navigation system, and it needs to establish time comparison links between international large time-frequency laboratories, and the operation mode is not flexible enough. This also leads to the shortcomings such as poor real-time performance and the difficulty of direct acquisition by the user, which restricts its application in the actual navigation and positioning.

Based on this, this section will construct a feasible method for monitoring time difference directly in the client using a multi-mode receiver fusion PPP method. First, analyze the concept and nature of GNSS time difference required by the actual navigation and positioning user.

\subsection{Concept of "pseudo-time difference" for navigation and positioning of users}

For the navigation and location users of the multimode receiver, the most important contribution of the system time difference is to reduce the number of receiver clock parameters to be estimated, and then reduce the necessary satellite observations. Taking GPS/GLONASS as an example, the receiver's clock error parameter of GPS system $\hat{t}_{r}^{G}$ and the receiver clock error parameter of GLONASS system $\hat{t}_{r}^{R}$, absorb the absolute device delay error $b$ and inter-frequency relative equipment delay error $\delta b$ on the respective system in addition to the real receiver clock difference $t_{r}^{G}$ and $t_{r}^{R}$ under its own time system. The specific formula expression is as follows [9]:

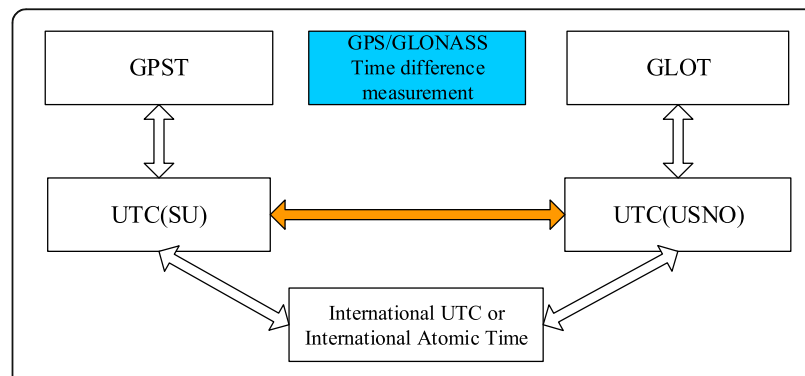

Fig. 1 Acquisition of time difference through GPS/GLONASS system time comparison 


$$
\begin{aligned}
& \hat{t}_{r}^{G}=t_{r}^{G}+b^{G}+\delta b^{G} \\
& \hat{t}_{r}^{R}=t_{r}^{R}+b^{R}+\delta b^{R}
\end{aligned}
$$

Subtract the left and right sides of (18) and (19) by two and it could be obtained that:

$$
\hat{t}_{\mathrm{sys}}=\hat{t}_{r}^{G}-\hat{t}_{r} R=t_{r}^{G}-t_{r}^{R}+\left(b^{G}-b^{R}\right)+\left(\delta b^{G}-\delta b^{R}\right)
$$

The time difference $\hat{t}_{\text {sys }}$ in the type (20) includes both the real GPS/GLONASS system time difference $t_{\text {sys }}\left(t_{\text {sys }}=t_{r}^{G}-t_{r}^{R}\right)$ and the poor delay of the GPS/GLONASS device $\left(b^{G}-b^{R}\right)+\left(\delta b^{G}-\delta b^{R}\right)$. The time difference of this property is called the "pseudo-time difference" of the receiver.

It can be seen that the difference between the real time difference $t_{\text {sys }}$ and pseudo-time delay difference $\hat{t}_{\text {sys }}$ is the equipment time delay $\left(b^{G}-b^{R}\right)+\left(\delta b^{G}-\delta b^{R}\right)$ between GPS/GLONASS system. If the equipment time delay signal of the GPS system and GLONASS system in the receiver is the same, then the pseudo-time difference is real-time difference; otherwise, the two are not equivalent.

\subsection{Acquisition of pseudo-time difference based on fusion PPP technology}

First, the observational equations of the simplified fusion PPP are listed as follows:

$$
\begin{aligned}
& P_{\mathrm{IF}}=\hat{\rho}+\mathrm{c} t_{r}+b_{P}+\delta b_{P}+\varepsilon\left(P_{\mathrm{IF}}\right) \\
& \Phi_{\mathrm{IF}}=\hat{\rho}+\mathrm{c} t_{r}+b_{\Phi}+\delta b_{\Phi}+N+\varepsilon\left(\Phi_{\mathrm{IF}}\right)
\end{aligned}
$$

Among them, $\hat{\rho}$ represents the geometric distance between the station and the satellite after the correction of the atmospheric error and so on. $t_{r}$ represents the receiver clock difference. The receiver's code and phase device time delay is divided into two parts: one is the fixed time delay on the frequency $b_{P}$ and $b_{\phi}$, the other is the relative device time delay between the frequency $\delta b_{P}$ and $\delta b_{\phi}$. For the phase observation, if the ambiguity parameter is a floating point solution, the fixed time delay $b_{\phi}$ and relative time delay $\delta b_{\phi}$ can be absorbed by the ambiguity parameter, and there is no need to solve in the actual navigation and location. For code observation, if the device delay is not corrected, it will be absorbed by the receiver clock error parameter, which will directly affect the absolute value of the receiver clock offset. Therefore, the absolute accuracy of the satellite clock difference is determined by the code observation value in the process of solving the actual satellite clock difference. The phase observation value determines the relative accuracy of the satellite clock difference calculation.

At present, IGS organizations provide three types of relative equipment delay correction products for code observations, also known as differential code deviation correction products. It should be noted that in addition to $\mathrm{DCB}$ corrections for satellites announced in DCB products, DCB corrections on stations are also announced. The measurement station DCB correction is often overlooked in GNSS positioning, mainly because it can be absorbed by the receiver clock skew and does not affect the actual navigation positioning solution. However, in time difference monitoring, this correction is very important. It directly affects the magnitude of the receiver clock difference and must be subtracted from the observation equation.

DCB products with IGS can correct relative device delays $\delta b_{P}$ between frequencies. However, absolute device delay $b_{P}$ cannot be eliminated. In other words, the time difference obtained by using the fusion PPP method is not a true time difference, but a pseudo-time difference containing the poor delay of the absolute equipment between systems, which can be expressed by the following formula:

$$
\bar{t}_{\mathrm{sys}}=t_{\mathrm{sys}}+\left(b_{P}^{G}-b_{P}^{R}\right)
$$

It can be seen from the above equation that when the absolute device delays of different navigation systems are the same, the fusion PPP gets the real system time difference sequence. In fact, the absolute device delays of different navigation systems in the same receiver are different, and the phase difference can range from a few nanoseconds to hundreds of nanoseconds.

\section{Experience}

In order to compare and verify the efficiency and accuracy of this algorithm, the following two types of examples are designed to verify it.

\subsection{Comparison of operational efficiency}

First, 1000 epoch observation data for the four navigation systems GPS, GLONASS, COMPASS, and Galileo were simulated. Each system has ten observation satellites per epoch. Satellite replacement does not occur during the observation period. The computational efficiency of multi-mode fusion PPP positioning based on traditional algorithm and parameter reduction is calculated respectively. Fusion static PPP location calculation of single-system, dual-system, three-system, and four-system is performed. The data processing carrier is based on a high-performance configuration computing computer. Both schemes use different numbers of GNSS systems for fusion PPP positioning. The computational time statistics needed to process 1000 epochs are shown in Fig. 3:

As can be seen from Fig. 2, with the increase in the number of GNSS fusion systems, the processing time of traditional fusion algorithms increases exponentially, and the computational burden increases with the same type. The parameter reduction algorithm proposed in this paper 


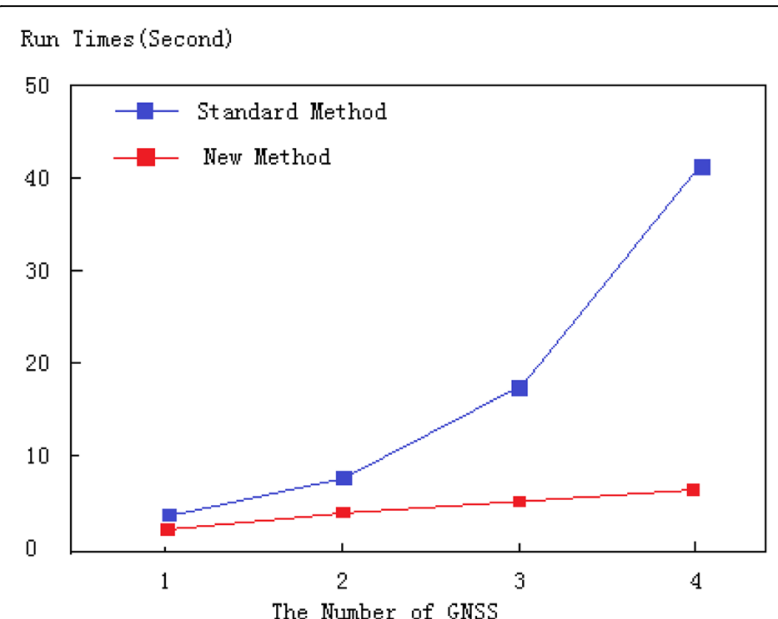

Fig. 2 Time information of multi-system fusion PPP processing carried out by two methods shows a linear increase in processing time. With the wide application of multi-mode systems, the computational efficiency of this algorithm is significantly higher than the traditional algorithm.

\subsection{Static and dynamic accuracy test of fusion positioning} In order to further verify the correctness of the fusion PPP location algorithm and the effectiveness of the adaptive factor, the measured GPS/GLONASS dual-system observation data is used for calculation and analysis. The data comes from the global IGS tracking and monitoring network, and 24 IGS stations equipped with GPS/GLONASS dual-mode receivers that are evenly distributed on the European continental plate are selected for static PPP calculation. The data sampling rate is $30 \mathrm{~s}$. The total observation period of data is $24 \mathrm{~h}$.

Two scenarios were designed for comparative analysis: case 1, fusion PPP positioning based on parameter reduction, and case 2, self-adaptive fusion PPP positioning based on the posttest difference. Using the coordinates of the stations posted on the IGS website as "true values," the deviation values of the positioning results of each station in $N, E$, and $U$ directions were calculated, and the overall RMS indicators corresponding to the deviation values of all stations were calculated. The statistics of deviations and RMS values of static PPP positioning results for specific experiments are shown in Fig. 3.

It can be seen from Fig. 4 that for a single-day solution, in the dual system fusion PPP positioning, the positioning accuracy on the plane can reach the millimeter level. The accuracy of the elevation direction is also maintained at about $1 \mathrm{~cm}$. This aspect shows good observation conditions at the IGS tracking station. On the other hand, it also illustrates the high-precision features of current PPP technology. Scenario 1 uses only equal

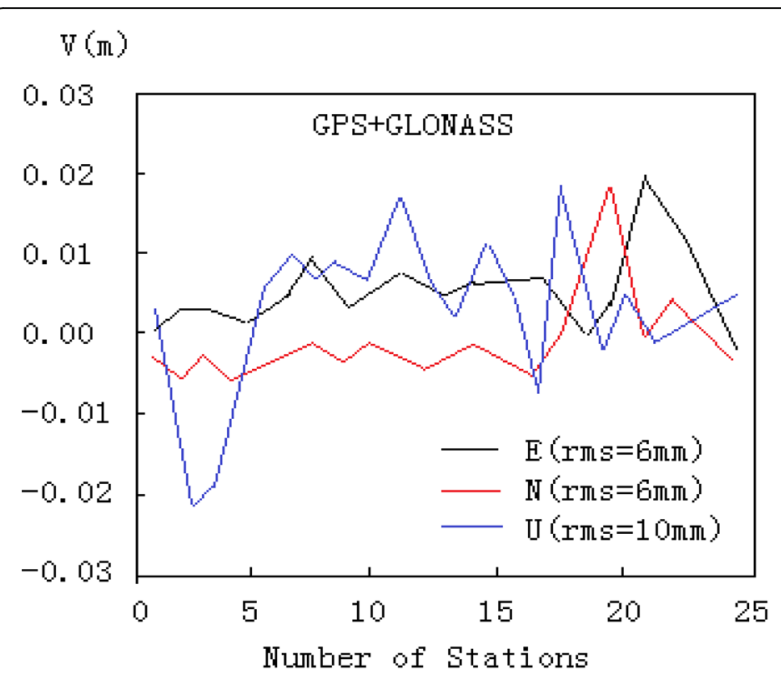

Fig. 3 Positioning result deviation and RMS value of case 1

weights to carry out the normal equation stacking and cannot reasonably assign the weight ratio relationship between GPS and GLONASS observations. The resulting fusion location results and accuracy lie between the positioning accuracy of the two single systems. The use of a posterior misalignment for adaptive fusion positioning (case 4) solves this problem better and has the best positioning accuracy.

In addition, in order to further verify the effectiveness and accuracy of the proposed algorithm in dynamic PPP location, a high-frequency IGS monitoring station equipped with a GPS/GLONASS dual-mode receiver was selected for dynamic PPP calculation. The data sampling rate is $1 \mathrm{~s}$, and the total data observation duration is $5 \mathrm{~h}$. Using the coordinates of the stations published on the IGS website as the "true value," the sequence of deviations in the $N, E$, and $U$

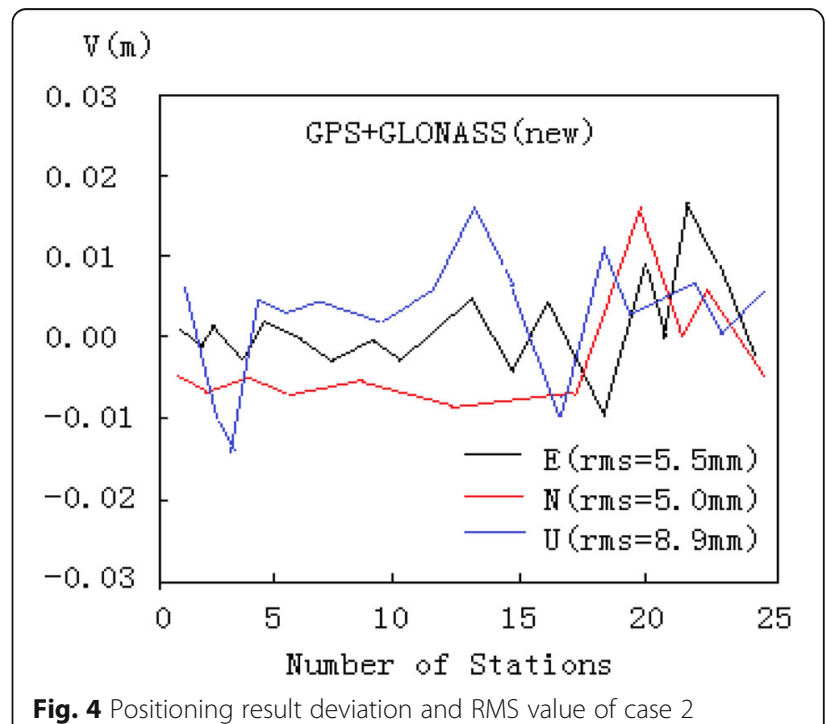




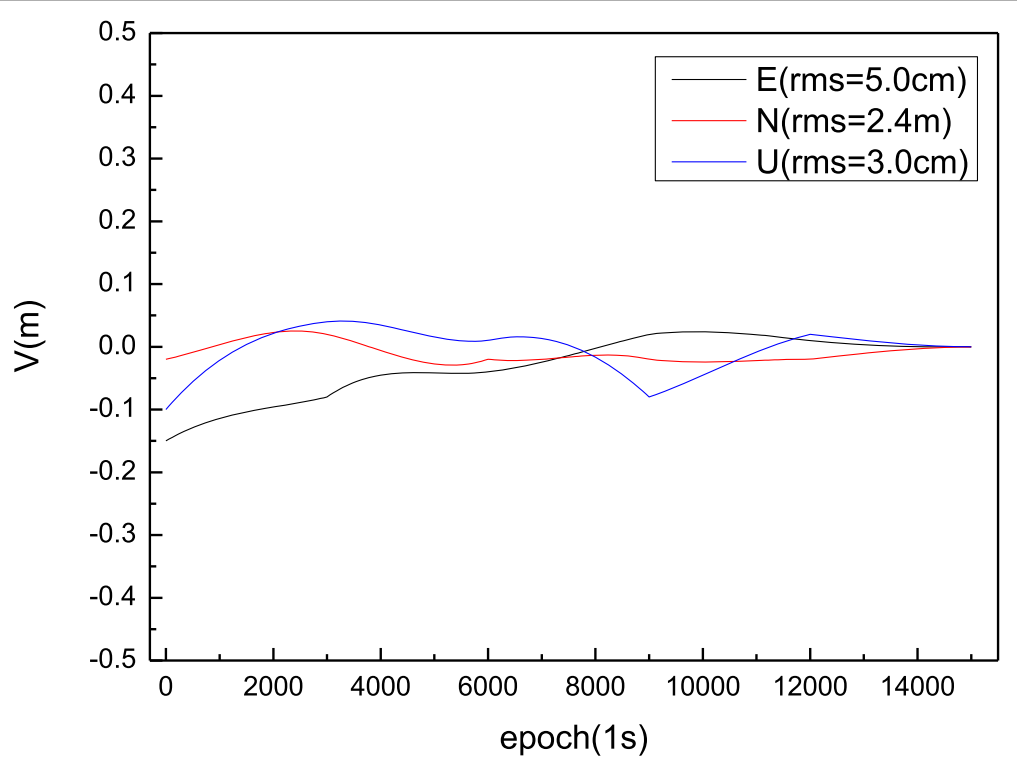

Fig. 5 Dynamic positioning deviation and RMS value of case 1

directions of the station's dynamic positioning results was calculated and the corresponding RMS values were calculated. The statistics of the dynamic PPP positioning deviation sequence and the RMS index value for specific experiments are shown in Fig. 5.

It can be seen from Fig. 6 that whether it is equal-value fusion PPP (case 1) or adaptive fusion PPP (case 2), the dynamic positioning accuracy is better than the single-system PPP positioning result. This shows that multi-system fusion positioning has significant advantages for improving the accuracy of dynamic positioning. When the observation conditions are good, the positioning accuracy of the two conditions is basically the same.

In order to further analyze the validity of case 2 in the presence of gross errors in observations, this article adds a phase error of $1 \mathrm{~m}$ to the first GPS satellite of each epoch during the 10,000th to 12,000th epoch of dynamic stations. The observations after 10,000 epochs were compared using case 1 and case 2 . The positioning results are shown in Fig. 7.

The change in the ratio of the adaptive factor of GLONASS to GPS of case 2 is shown in the following diagram.

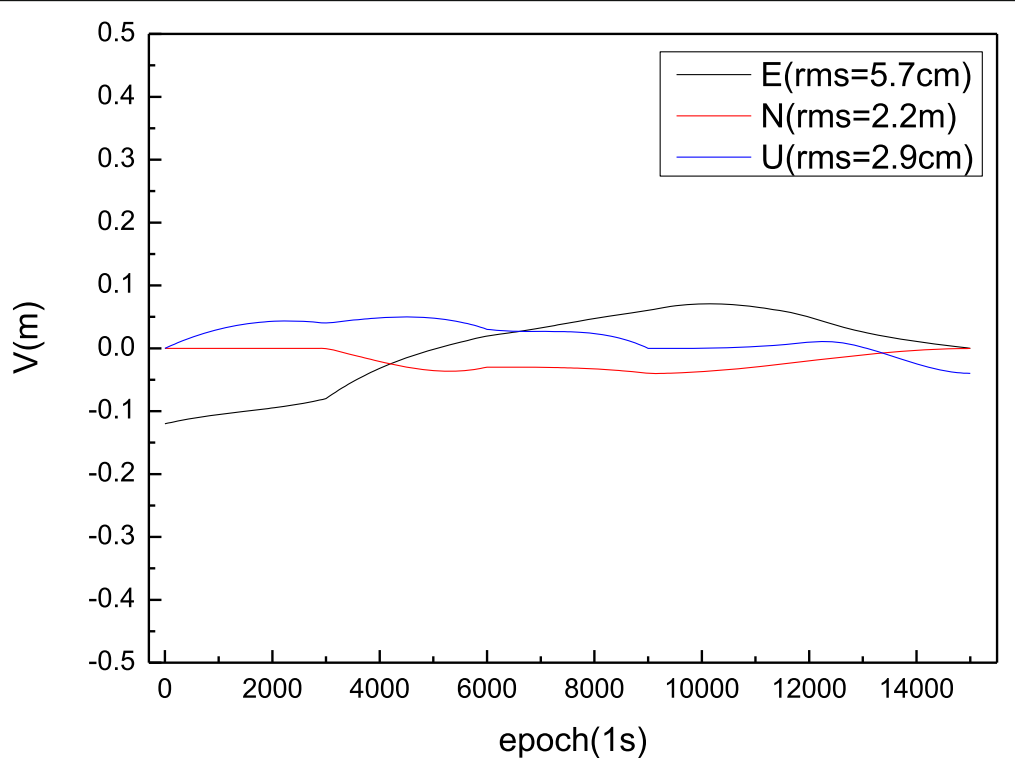

Fig. 6 Dynamic positioning deviation and RMS value of case 2 


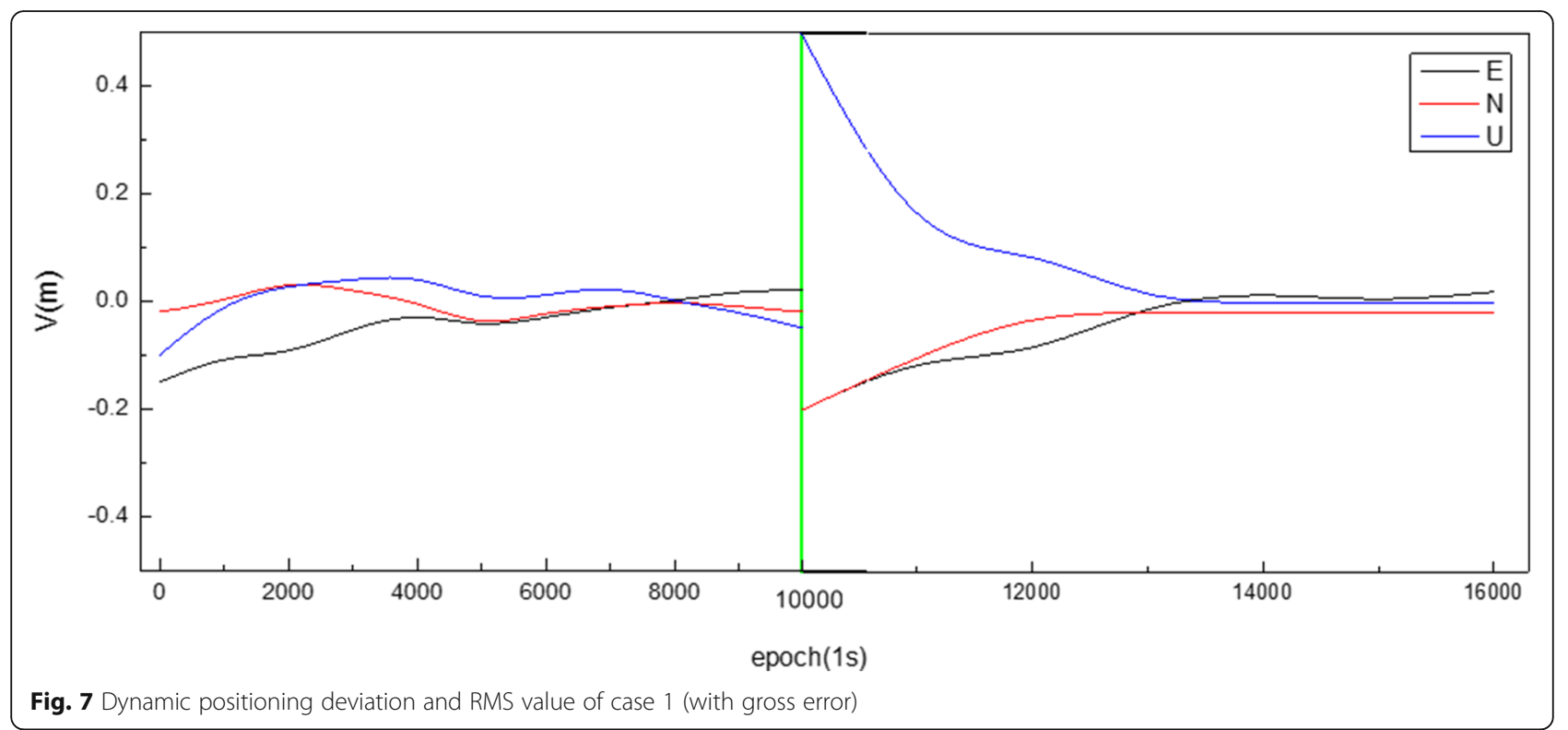

From Figs. 7, 8, and 9, it can be seen that when there is a rough difference between the observed values of a single system, the location results of case 1 are seriously affected, and the situation 2 is less affected. The reason is that when a single system experiences a gross error in observations, the resulting single-system test posterior error will gradually become larger, so that the adaptive factor of case 2 will be automatically adjusted to redistribute the fusion weight ratio relationship of different systems and reduce the gross error ratio and reduce the contribution of system normal equations with gross errors to achieve the purpose of suppressing gross errors.

\subsection{Short term stability of fusion PPP pseudo-time difference results}

In this section, the data of 14 GPS/GLONASS observation stations in the IGS continuous tracking station network in the European region were uniformly selected for analysis [10]. The data acquisition time is March 22, 2012 , the data sampling rate is $30 \mathrm{~s}$, and the total observation time is $24 \mathrm{~h}$. The satellite orbit and clock difference products are derived from the IAG products provided by the MCC Analysis Center under the IGS organization. The GPS/GLONASS orbit of IAG products has been classified into the ITRF framework, and the satellite clock difference is kept under the respective

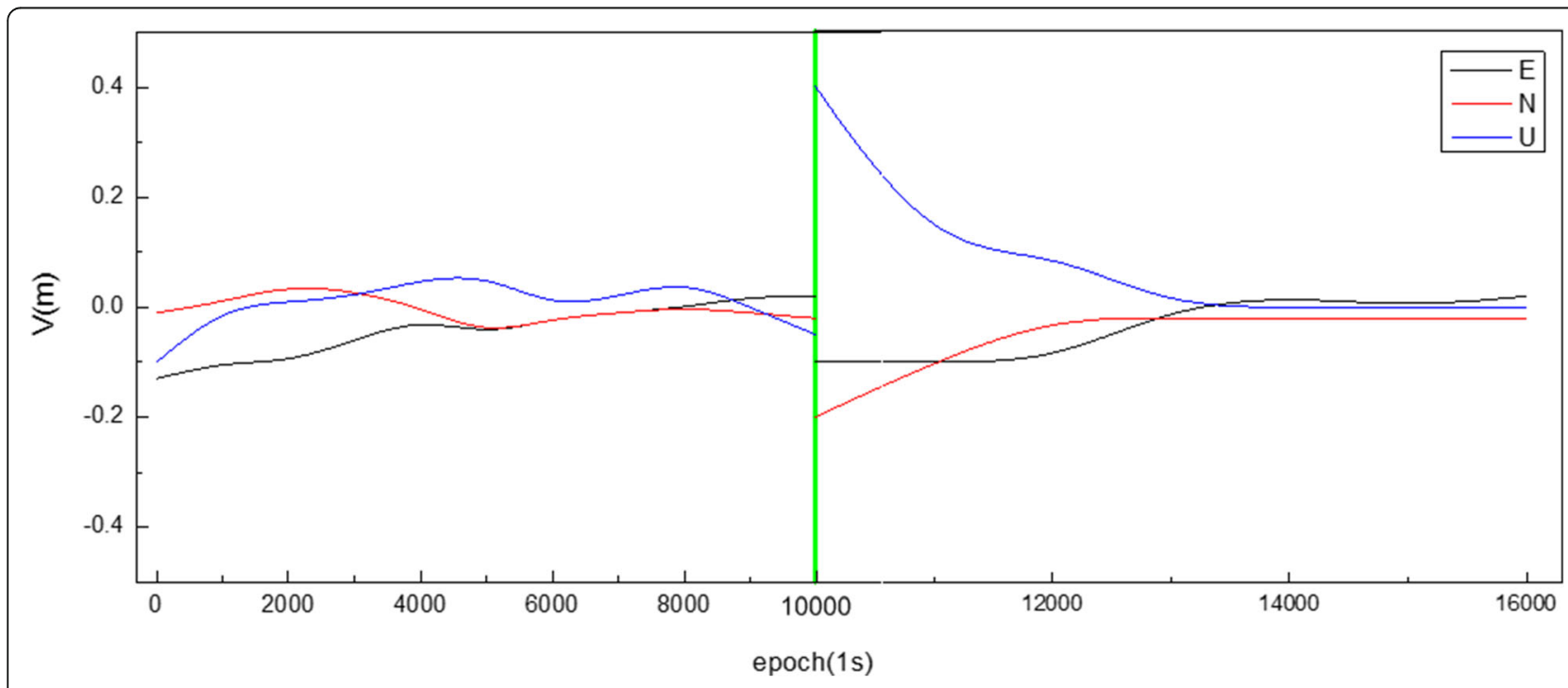

Fig. 8 Dynamic positioning deviation and RMS value of case 2 (with gross error) 


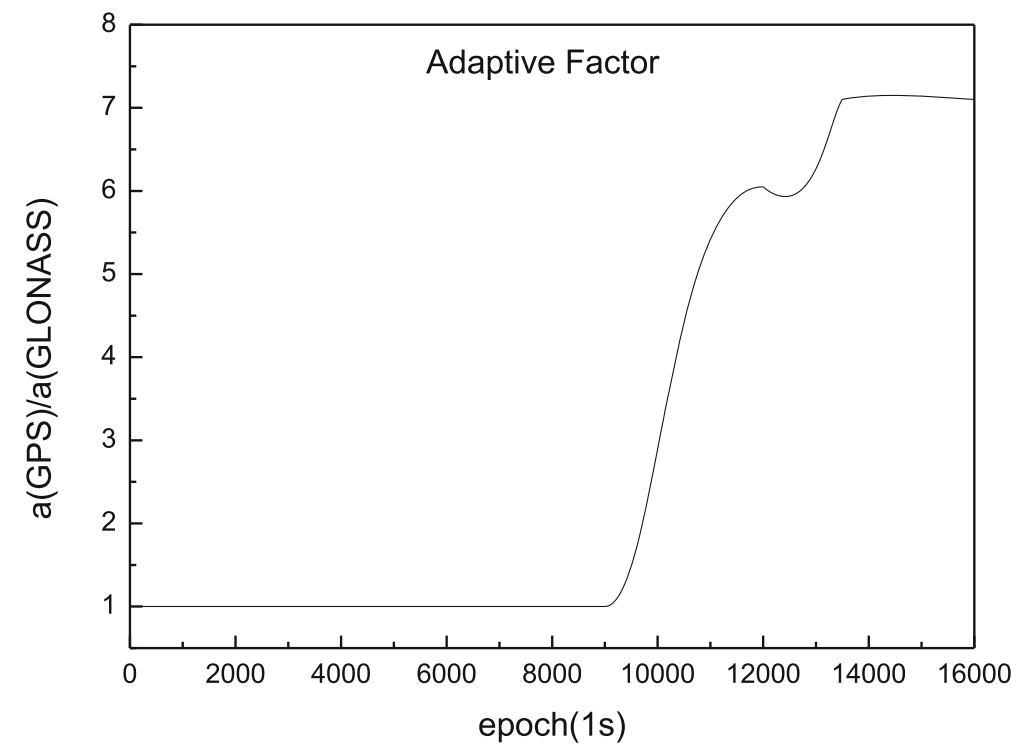

Fig. 9 Change of adaptive factor ratio in case 2

time system. The sampling rate of the track product is $15 \mathrm{~min}$, and the sampling rate of the clock difference product is $5 \mathrm{~min}$. All stations are equipped with dual frequency dual mode GNSS receivers. In order to compare the difference in the result of the time difference between the different receiver types, the station name and the receiver type information are listed, as shown in Table 1:

As you can see from Table 1, there are six kinds of receiver types in the 13 selected stations. There are six stations in which the LEICA receiver is equipped. Four stations are equipped with TRIMBLE receivers. The other types of receivers have only one station configuration each. The mean and standard deviation of the time difference sequence on all stations are counted, as shown in the Table 2:

The standard deviation index of the time difference sequence of different stations is compared, as shown in Fig. 10.

Table 1 Station name and the receiver type information

\begin{tabular}{llll}
\hline Station name & Receiver type & Station name & Receiver type \\
\hline CAGZ & TPS E_GGD & PENC & LEICA CR $\times 1200$ \\
ONSA & JPS E_GGD & GANP & TRIMBLE NETR8 \\
GLSV & NOV OEMV3 & GRAS & TRIMBLE NETR5 \\
HERT & LEICA CR $\times 1200$ & TLSE & TRIMBLE NETR9 \\
JOZ2 & LEICA CR $\times 1200$ & ZIM2 & TRIMBLE NETR5 \\
LAMA & LEICA CR $\times 1200$ & WTZZ & JAVAD TRE_G3TH \\
MATE & LEICA CR $\times 1200$ & & \\
\hline
\end{tabular}

The Allen variance is used to calculate the single day frequency stability index of time difference sequences of the different station, such as 11 .

As can be seen from the graph in Figs. 10 and 11, the result of the time difference sequence obtained by the same type of receiver is relatively close, and the absolute difference of the time difference is less than $20 \mathrm{~ns}$. This is mainly due to the close proximity of the absolute hardware delay of the same type of receiver, so the time difference sequence is relatively close. There is a large system error due to the difference of absolute hardware delay between different receivers[11]. The maximum

Table 2 Mean and standard deviation of the time difference sequence (unit: nanosecond)

\begin{tabular}{llll}
\hline Station name & Receiver type & Mean & Standard deviation \\
\hline CAGZ & TPS E_GGD & -448.257 & 0.423 \\
ONSA & JPS E_GGD & -464.486 & 0.286 \\
GLSV & NOV OEMV3 & -348.679 & 1.123 \\
HERT & LEICA CR $\times 1200$ & -337.857 & 0.546 \\
JOZ2 & LEICA CR $\times 1200$ & -353.536 & 0.475 \\
LAMA & LEICA CR $\times 1200$ & -345.758 & 0.389 \\
MATE & LEICA CR $\times 1200$ & -333.689 & 0.732 \\
PENC & LEICA CR $\times 1200$ & -345.785 & 0.598 \\
GANP & TRIMBLE NETR8 & -374.537 & 0.694 \\
GRAS & TRIMBLE NETR5 & -375.974 & 0.645 \\
TLSE & TRIMBLE NETR9 & -364.876 & 0.432 \\
ZIM2 & TRIMBLE NETR5 & -384.438 & 0.564 \\
WTZZ & JAVAD TRE_G3TH & -398.343 & 0.654 \\
\hline
\end{tabular}




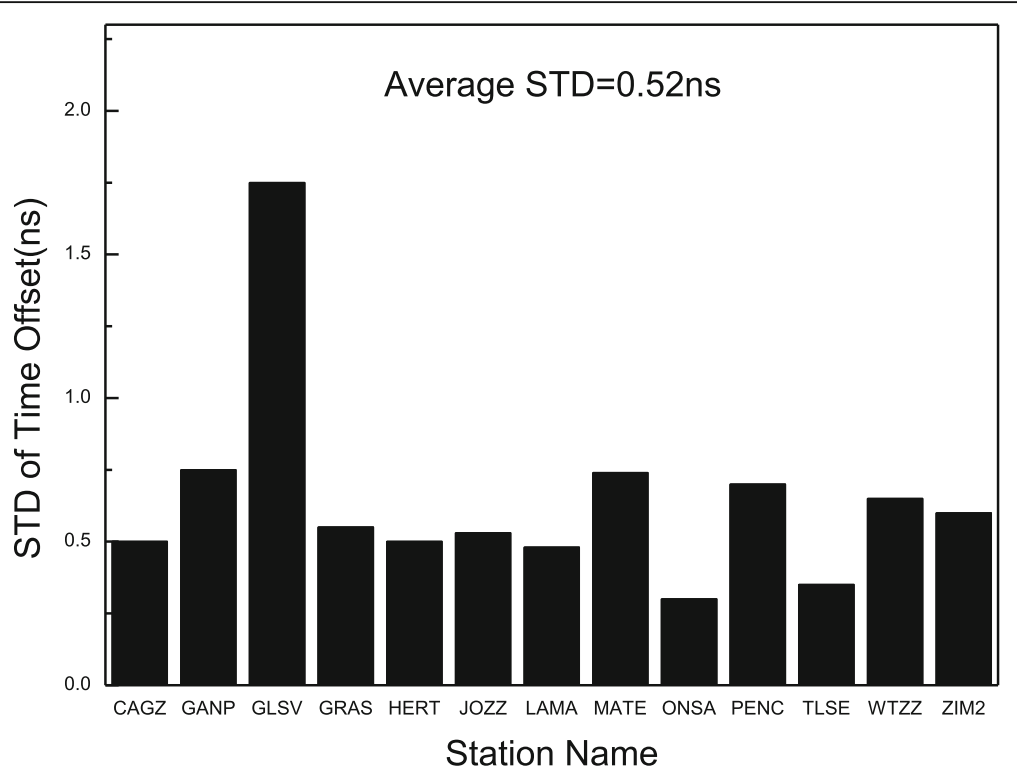

Fig. 10 Standard deviation statistics for time difference sequences of different stations

mean time difference is more than $130 \mathrm{~ns}$. The result of the time difference sequence on all stations is very stable in 1 day. Most of the standard deviation of the time difference sequence is better than $1 \mathrm{~ns}$, and the average standard deviation is only $0.52 \mathrm{~ns}$. This stable characteristic provides a good premise for the follow-up time difference prediction and the application in navigation and positioning[12]. The single-day frequency stability analysis of the TDOA sequence shows that the single day stability index of the TDOA sequence obtained by the fusion PPP is about the magnitude of $10^{-14}$, and the average frequency stability is $3.0 \times 10^{-14}$. This shows that it is feasible to use the fusion PPP method to monitor the time difference of the system from the precision[13].

\section{Results and discussion}

The traditional multi-mode GNSS fusion precise singlepoint positioning algorithm has the disadvantages of low efficiency and difficulty in satisfying the demand for high-accuracy real-time and high-frequency data[14]. To solve the above problems, this paper proposes an adaptive GNSS fusion PPP algorithm based on parameter

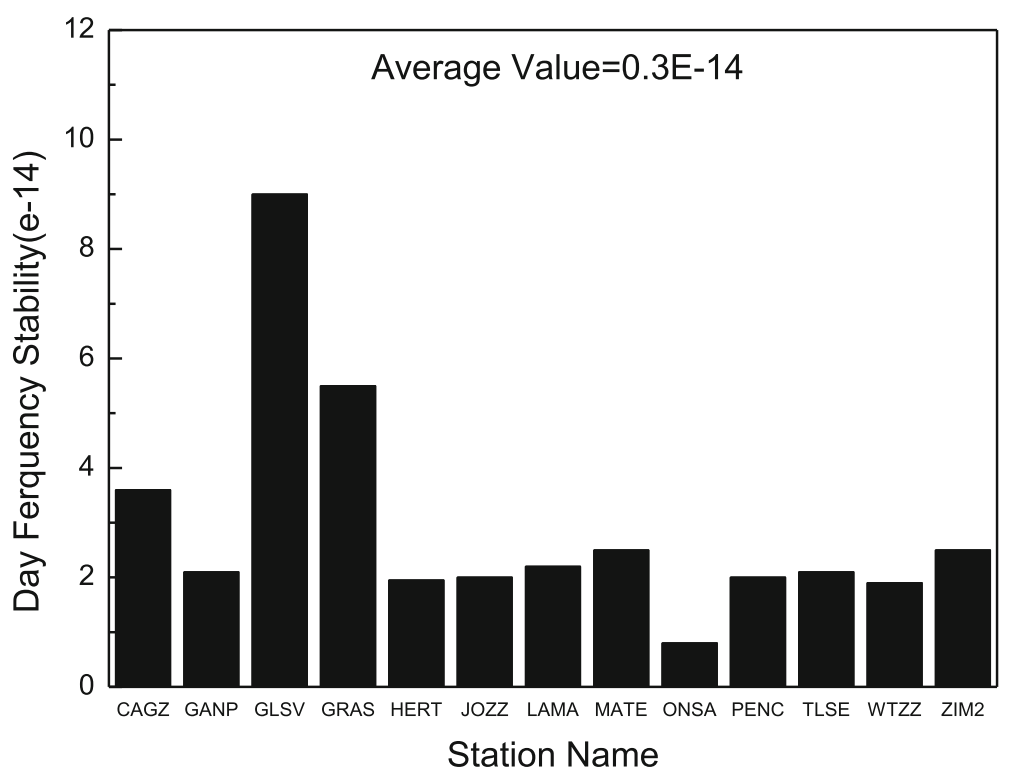

Fig. 11 Frequency stability index of the time difference sequence of different stations 
equivalence reduction. With the increase of the number of fusion systems, the computing load of traditional algorithms is exponentially increasing. The new algorithm improves it to a linear growth, which greatly improves the computational efficiency. The actual example verifies the effectiveness and accuracy of the improved algorithm. Secondly, this paper also studies the feasibility and algorithm flow of GNSS time difference monitoring using multi-system fusion PPP algorithm at the user end. Pseudo-time difference monitoring and forecasting work has more important application value for navigation and positioning users[15]. The real system time difference has no practical significance for navigation users.

\begin{abstract}
Abbreviations
GLONASS: GLObalnaya NAvigatsionnaya Sputnikovaya Sistema; GNSS: Global Navigation Satellite System; GPS: Global Position System; PPP: Point-to-Point Protocol; UTC: United Technology Corporation

Funding

Technology Platform Project of Guangdong Provincial Department of Education (2017GKTSCX104), Plan Project of Guangdong Science and Technology (2014A010103002)
\end{abstract}

\section{Availability of data and materials}

The datasets used and/or analysed during the current study are available from the corresponding author on reasonable request.

\section{Authors' contributions}

ZZ carried out the time difference detection algorithm studies, participated in the sequence alignment and drafted the manuscript. ZX and HC carried out the Data test. JY and YL participated in the sequence alignment. WZ participated in the design of the study and performed the statistical analysis. YC conceived of the study and participated in its design and coordination. All authors read and approved the final manuscript.

\section{Authors' information}

Ziwen Zhang, Male, Postdoctoral, 1987-11-25, Guangdong University of Technology.

Major: High-precision satellite positioning, radar interferometry, deep learning.

Jun Yang, Male, postgraduate, Chongqing Vocational College of Transportation.

Main research direction: high-precision satellite positioning, radar interferometry, deep learning.

Yijun Liu, Male, Professor, Guangdong University of Technology.

Major: High-precision satellite positioning, deep learning.

Zuoteng Xu, Male, Master student, Guangdong University of Technology,

Communication Engineering.

Main research direction: Deep learning, computer graphics processing. Haowen Chen, Male, Master student, Guangdong University of Technology. Main research direction: Deep learning, computer graphics processing. Yuqiang Chen, Male, Professor, 1983-07-13, Gongguan Polytechnic. Main research direction: Satellite high-precision positioning, artificial intelligence.

\section{Competing interests}

The authors declare that they have no competing interests.

\section{Publisher's Note}

Springer Nature remains neutral with regard to jurisdictional claims in published maps and institutional affiliations.

\section{Author details}

${ }^{1}$ Chongqing Vocational College of Transportation, Chongqing 402247, China.

${ }^{2}$ School of Information Engineering, Guangdong University of Technology,
Guangzhou 510006, China. ${ }^{3}$ Dongguan Polytechnic, Dongguan 523000, China.

Received: 29 December 2018 Accepted: 26 March 2019

Published online: 07 May 2019

\section{References}

1. Z. Hongping, Z. Gao, X. Niu, et al., Research on GPS precise point positioning with un-differential and un-combined observations Geomatics \& Information Science of Wuhan University 28(6), 217-221 (2013)

2. W. Jiang, Y. Li, C. Rizos, An optimal data fusion algorithm based on the triple integration of PPP-GNSS, INS and terrestrial ranging system (2015)

3. Q. Zhao, B. Sun, Z. Dai, et al., Real-time detection and repair of cycle slips in triple-frequency GNSS measurements. GPS Solutions 19(3), 381-391 (2015)

4. H. Zhang, Z. Gao, M. Ge, et al., On the convergence of ionospheric constrained precise point positioning (IC-PPP) based on undifferential uncombined raw GNSS observations. Sensors 13(11), 15708-15725 (2013)

5. Q. Zhang, S. Tan, S. Yang, GNSS system time bias estimation based on PPP. J. Geom. 41(01), 27-30 (2016)

6. C. Liang, H.U. Zhigang, C. Geng, et al., Study on a high-frequency multiGNSS real-time precise clock estimation algorithm and application in GNSS augment system. Acta Geodaetica Et Cartographica Sinica. 45(S2), 12-21 (2016)

7. M. Ge, G. Gendt, M. Rothacher, et al., Resolution of GPS carrier-phase ambiguities in precise point positioning (PPP) with daily observations. J. Geod. 82(7), 389-399 (2007)

8. M.R. Kaloop, M. Rabah, Time and frequency domains response analyses of April 2015 Greece's earthquake in the Nile Delta based on GNSS-PPP. Arab. J. Geosci. 9(4), 1-13 (2016)

9. Tobías G, Calle J D, Navarro P, et al. magicGNSS' Real-Time POD and PPP Multi-GNSS Service. 2014

10. G. Huang, Y. Yang, C. Liu, et al., GNSS precise point positioning algorithm based on parameter equivalent reduction principle. Lect. Notes. Elect. Eng 244, 449-469 (2013)

11. N. Noomwongs, R. Thitipatanapong, S. Chantranuwathana, et al., Driver behavior detection based on multi-GNSS precise point positioning technology. Appl. Mech. Mater 619, 327-331 (2014)

12. G. Huang, Q. Zhang, Real-time estimation of satellite clock offset using adaptively robust Kalman filter with classified adaptive factors. GPS Solution (2012). https://doi.org/10.1007/s10291-012-0254-z

13. N. Abdi, A.A. Ardalan, R. Karimi, et al., Performance assessment of multiGNSS real-time PPP over Iran. Adv. Space Res. 59(12), 117-123 (2017)

14. U. Weinbach, S. Schön, GNSS receiver clock modeling when using highprecision oscillators and its impact on PPP. Adv. Space Res. 47(2), 229-238 (2011)

15. J.H. Zhang, H.B. Yuan, S.W. Dong, et al., Prediction of GNSS time difference based on combination of Grey model and quadratic polynomial model. J Time Freq 37(04), 199-205 (2014)

\section{Submit your manuscript to a SpringerOpen ${ }^{\circ}$ journal and benefit from:}

- Convenient online submission

- Rigorous peer review

- Open access: articles freely available online

High visibility within the field

- Retaining the copyright to your article

Submit your next manuscript at $>$ springeropen.com 